\title{
Chapter 14. The Propagation of Online Rumours Slandering Multinational Companies in Egypt
}

\author{
By Mona Shehata
}

\section{Introduction}

In competitive, changing and unpredictable environments, organisations can easily become victims of multiple rumours which spread on digital platforms and cause a lot of hazards. Colas (2005, p. 21) states that "Rumour is one of the oldest threats that an economic actor can face". Today, the spread of online rumours has become more ferocious and has different forms (photos, videos, graphics and visual signs) that amplify the attention of Internet users. These new forms of e-rumours are embarking on a multicultural orientation that requires organisations to adapt their communication strategies accordingly; by gaining knowledge on the societal and cultural aspects where they operate. Milliot (2016, p. 134) argues that "the cultural context therefore has a determining influence on the conception and implementation of economic intelligence". Using the term context, according to Hall's approach (1976), will allow us to comprehend the cultural impact on the diffusion and interpretation of internet users' interaction during the spread a specific rumour in the Egyptian context. It seems relevant to study in-depth the commercial rumour propagation to equip multinational companies to face these new challenges proactively and to keep their ereputation. Thus, the analysis is based on a three-level dynamics (micro, meso and macro environments).

To outline the nature of commercial rumours in the Egyptian context, three concrete case studies belonging to three different economic sectors were executed as follows: politics (Orange), economy (Pampers) and health (Danone). The research question that this chapter tries to answer can then be posed as follows: Are there distinct socio-cultural factors affecting the mechanism for spreading commercial rumours on Facebook in Egypt?

This general issue raises three sub questions:

- Q 1: What are the factors affecting rumours dissemination on Facebook?

- Q 2: What is the role of digital platforms, including Facebook, in the process of disseminating rumours?

- Q 3: To what extent can a country's cultural dimensions be considered as a barrier or leverage to the spread of rumours on social media?

To answer these questions, this chapter is designed of five parts.

1- The definition of a multi-level analysis grid from a literature review base.

2- The pluralism of the method adopted.

3- A detailed overview of the three case studies selected.

4- The main findings and the analysis of the cases.

5- Conclusion.

\section{A Multi-Level Analysis Grid}

The analysis is carried out on the micro, meso- and macro-environmental levels to determine the variables from the field study conducted in Egypt which acts as dissemination vectors. The delivery of these three levels will make it possible to assess the dynamics that ensure the creation, circulation, propagation, survival and death of rumours. Taking Difonzo and Bordia (2007, p. 283) 
analysis into consideration, we find that these three levels are mentioned in their study and it states that "real-life rumours (transmission, belief, distortion) almost certainly involve multi-level dynamic processes - micro, meso and macro".

To begin with the micro-environmental level which focuses on the mechanism of spreading rumours among Internet users in Egypt. In this section, we pinpoint the cultural dimensions that are associated with Internet users to find relevant answers to the sub-question Q1, and the researcher analyses four variables related to the dissemination mechanism at the micro level:

- The first variable focuses on the nature of the source that starts the rumour. Here we analyse the nature and type of profile on social media.

- The second is related to the timing and frequency of diffusing a rumour. According to Ploux (2001), the way rumours circulate is influenced by the gap between the scarcity of information and people's thirst for information. This may explain the variation in the frequency of rumour transmission to fill an information gap.

- The third variable is the trust and beliefs of "famous" people who play an undeniable role in spreading rumours if involved in the propagation process. In the field study, it is observed that a verified account of celebrities for example - in the course of propagation of a rumour - intensifies its effect by boosting the sharing action of the rumour due to the confidence and trust that Internet users have in them.

-The fourth and last variable, tackles the Internet users at the micro level, and it is related to conducting a sentiment and semantic analysis of their feelings as they share a rumour. Through this analysis, it is detected that some Internet users write comments on the original Facebook post before sharing it on their wall as a means of expressing themselves. These added messages represent a form of over-specification (Rouquette 1979 and 1975). They play the role of augmenting the spread of rumours, thus reinforcing the basic functionalities of digital platforms such as like, comment and share. According to Milliot's study (2016, p. 135 adapted from Hall, 1976), Egypt - situated amongst North African countries - is considered as a high cultural context, a country where cultural framework conditions communication schemes. Milliot (2016, p. 135) adds that: "The messages emitted in these cultures, characterized by a highly developed sense of community, are generally interwoven and implicit. One must analyse and decrypt them, according to a particular logic, to comprehend their true meaning".

The meso-environmental level is used to answer sub-question Q2. To conduct this, we analyse Facebook's features to find out how they can leverage the disseminating of rumours. In this respect, Alloing (2013, p. 134) argues that: "This production of information inherent to the functionalities of the socio-technical structures specific to the Web 2.0 allows it to develop new forms of relationships, or even to emancipate itself by contributing to the construction of knowledge and by participating in certain collective actions". From a meso-environmental perspective, we have identified four variables relevant to our field study that affect the spread of rumours and related to the functionality of digital social networks. 
- First, it is noted that rumours can be manifested in many forms: photos, videos, written statutes or even URL links (Uniform Resource Locator). Therefore, in each case, we will analyse this variable to determine if there is a repetitive form in all cases.

- The second variable concerns the affective metrics (example: likes, comments and sharing) provided by the Facebook platform. According to Kim and Yang (2017, p.447), "Facebook behaviours also fall into discrete levels. First, like is the lowest. Like requires less commitment than others do. While a click is enough for like, comment and share need additional actions that ask extra commitment or cognitive effort. Second, share may be a higher level than comment [...] On the other hand, when sharing a post; the post not only appears on News Feed but also goes to user's profile page, suggesting that the shared post constitutes a part of user's self-presentation".

- The third variable, linked to the specific characteristics of digital platforms, lies in the presence of transposable signs and hashtags in the path of rumours (either the initial content or the exchanges between Internet users). Transposable signs are defined by Le Béchec and Boullier (2014, p. 124) as "elementary semiotic forms of the territory that circulate easily on the web. This circulation and transposition produce a new version of what a regional scale is by making it visible and portable". The hashtag is defined according to Bruns and Burgess $(2011$, p. 1) as "a short keyword, prefixed with the hash symbol '\#' - as a means of coordinating a distributed discussion between more or less large groups of users, who do not need to be connected through existing 'follower' networks".

- The fourth variable is related to the traceability feature provided by the ability of digital platforms to archive old exchanged publications (the memory of the Web). Le Béchec (2010, p. 345) highlights that the memory of the Web of which the social media are a part of, is "a medium and a repository of memory". This dimension ensures that rumours survive on the platforms leaving behind digital traceability that helped us find and collect some data related to each case.

The macro-environmental level, which answers sub-question Q3, also includes four variables.

- First, we underline the important topics and themes related to the cultural context. The derived values from each topic are crucial for studying spreading a rumour.

- Second, we analyse the cultural features translated by the language and semantics used during the interactions between Internet users.

- Third, we extend the analysis to detect clusters formation. According to Latané's (1996) theory of dynamic social impact (DSIT), some cultural elements are more likely to spread and selforganise (i.e., to regroup, correlate and merge) than others.

- The last variable examines the behaviour of multinational companies that are victims of rumours. They show these behaviours in unofficial and/or official debunking statement to counteract the rumours.

It is important to mention that Shibutani's (1966) study inspired this analysis, which incorporates the notion of a situation into the definition of the circulation of a rumour (1966, p. 8-9). He also underlines a collective sense-making explaining that the circulation of a rumour is a transaction inside a group. Shibutani (1966, p. 17) explains that this allows rumours to aggregate all the 
understandings distributed within the community. Rumours are attempts to raise collective awareness. In this perspective, Shibutani (1966) considers the degree of emotional arousal that a rumour can trigger (cited by Brodin and Roux, 1990, p. 53).

\section{The Method of Data Collection on Facebook}

The choice of the Facebook platform to study the spread of rumour in Egypt was not selected randomly but was linked to two reasons. Firstly, Facebook is the most used platform in Egypt (72.12\% compared to $22.17 \%$ for YouTube and 2.96\% for Twitter in September 2019) ${ }^{1}$. Moreover, the total number of active Egyptian Facebook users reached 41,930,000 (or 40.9\% of the total population $)^{2}$. Second, $81 \%$ of the users in Egypt claim to have witnessed false information on the platform according to a study conducted by Ipsos Global Survey (p. 37 of the report) ${ }^{3}$. To collect data on exchanges between Internet users, we conducted a virtual ethnography (netnography). According to Kozinets (2002, p.2) "netnography, or ethnography on the Internet, is a new qualitative research method that adapts ethnographic research techniques to the study of cultures and communities emerging through computer-mediated communications".

To concretize this study, the multiple case study method is used, it involves "intensive study of a unit for the purpose of understanding a larger class of (similar) unit observed at a single point in time or over some delimited period of time" (Gerring, 2004, p. 342). The multiple case (multi-case) approach is relevant for "giving a broader picture of a complex phenomenon" (Stake, 2006) and for comparing different realities in different industries.

\section{The Presentation of the Three Case Studies}

\subsection{Danone Egypt}

\section{Rumour: «Danone yoghurts contain pork gelatin »}

\section{Presentation of the case}

In 2016, the dairy company Danone was hit by a rumour on social networks in Egypt accusing it of using gelatin from pork skin in the manufacture of its yoghurts.

\section{The origin of the rumour}

The theme of the rumour concerns religious morals and beliefs of Muslim communities since the consumption of pork and all its derivatives is prohibited in Islam. In 2016 the rumour started in

\footnotetext{
1 According to the statistics of the study of the stat counter site. Link to the study: https://gs.statcounter.com/social-mediastats/all/egypt/2019 retrieved on October 30, 2019.

${ }^{2}$ Study conducted statistically by Napoleon Cat, link to https://napoleoncat.com/stats/facebook-users-in-egypt/2019/09 retrieved on October 30, 2019.

${ }^{3}$ Survey conducted by Ipsos on behalf of the Centre for International Governance Innovation ("CIGI") between 21 December 2018 and 10 February 2019. The survey was conducted in 25 economies - Australia, Brazil, Canada, China, Egypt, France, Germany, Great Britain, Hong Kong (China), India, Indonesia, Italy, Japan, Kenya, Mexico, Nigeria, Pakistan, Poland, Russia, South Africa, Republic of Korea, Sweden, Tunisia, Turkey and the United States - and involved 25,229 Internet users. Available on 2019 CIGIIpsos Global Survey - Part 3 Social Media, Fake News \& Algorithms. Source available on: https://www.cigionline.org/sites/default/files/documents/2019\%20CIGI-

Ipsos \%20Global\%20Survey\%20\%20Part\%203\%20Social\%20Media\%2C\%20Fake\%20News\%20\%26\%20Algorithms.pdf retrieved on June 17, 2019
} 
Egypt, when it was spread by a public page on Facebook called "Alexandria 24 Hours". This is an Egyptian tabloid-type page that shared the rumour as a photo that was widely circulated. It contained the pots of Danone yoghurt with a photo of a pig and a brief narrative of a text written in Arabic. This text begins with a religious expression generally used in times of disappointment to generate feelings of empathy with the Muslim community that has been deceived and causes resentment against Danone. It ends with a call for action to boycott the products (the translation of the text in the footnote). In addition, an indexed hashtag in Arabic: زبادي_دانون (\#yaourt_Danone) has been added to the publications.

It is important to mention that the roots of the rumour date back to 15 September 2012 when it first appeared on the Web in a video 4 on YouTube entitled: زبادى دانون DANONE ودانيت DANETTE هل 5هما جيلاتين الخنزير؟ (Translation: Do Danone and Danette yoghurts contain pork gelatin). This video was downloaded by a religious page called HolyQuranbyHosary. The source is a YouTube channel with $1.38 \mathrm{~K}$ subscribers. This page broadcasted the video in French, but with Arabic subtitles, with an intention to target a Franco-Arabic community. The total number of views on YouTube exceeded 100,000, exactly 115,960 views, 26 comments from 2012 to 2019 and 343 likes until October 17, 2019. The rumour continued its life cycle on Facebook and Twitter from 2012 until September 2018 through international interactions among Internet users.

By observing the timestamp of the dissemination of publications that spread the rumour over the years, we can notice a cyclical diffusion. For example, in Egypt the rumour appeared just before the month of Ramadan, known as the yogurt consuming season. The rumour on Facebook lasted two years and five months.

The reaction of Internet users on Facebook was mainly sharing this rumour more than liking it or commenting on it. Some messages written in Arabic were added to these shares by Internet users, contained religious expressions such as "Allah is sufficient for me, he is the best protector" to express their dissatisfaction.

\section{Rumour management/debunking?}

The public page on Facebook called Da begad played the role of fact-checking in Egypt and denied the rumour three days after it appeared on Facebook ${ }^{6}$. Danone Company has not published anything and has not reacted.

\subsection{Pampers Egypt}

\section{Rumour: «Pampers prices are rising in Egypt after applying the new VAT »}

\footnotetext{
${ }^{4}$ Link to the video, https://www.youtube.com/watch?v=Ew65Tds3a_o\&feature=youtube_gdata_player retrieved on October 17, 2019.

${ }^{5}$ The title was scripted using Arabic and English words on the original video on YouTube.

6 Link of the debunking published on Facebook by "Da begad". retrieved on 30 , April 2018 https://www.facebook.com/DaBegad/photos/a.178588762292398/644216762396260/?type=3\&theater
} 


\section{Presentation of the case}

This rumour was launched on the 19th of July 2016 on the Egyptian social media, on the occasion of government announcements concerning economic reforms aimed at changing the VAT of some goods and services in Egypt. A public page called "Porto Tora for you"7 on Facebook has published a photo containing Arabic text and hashtags indexed by a fake account under the name of "Nesma Alaa". We analysed the Social media platforms, including Facebook, and found that this publication was the first to disseminate this topic. We therefore, believe that it was what triggered the rumour. The name of the page already reveals that it is a page opposing the regime, because it holds the name of a prison in Egypt called "Tora". The translation of the name of the public page is "Porto Tora prison is made for you" implicitly to ironically mean that all those who oppose the regime will end up in prison.

On the occasion of the new law, the rumour found a favourable ground to emerge and to trigger dismay in society even though these legislations had not yet been made official. It's also important to mention that Procter \& Gamble effectively raised Pampers prices six months after the rumour spread in January 2017 following the devaluation of the Egyptian pound, which caused exchange rate fluctuations.

In the content of the rumour, we note two hashtags indexed in Arabic with an Egyptian dialect: \#Taxe_added and \#Piss_on_Egypt_my_baby. The public page also added a sarcastic comment to the photo: "even Pampers + laughing emoticon". We suggest that the insertion of a laughing emoji along sarcastic hashtags can capture the attention of Internet users and make the rumour more engaging.

\section{Translation of the publication:}

The Pampers box costs 97 L.E. two weeks ago... Today, it costs 158 L.E ${ }^{8}$.

\#Added_tax

\#Piss_on_Egypt_my_baby.

It was noticed on Facebook that the most common reaction of Egyptian Internet users was the likes, not the sharing of the publication of the rumour. Messages written in Arabic, which were added to the shares by Internet users, were also analysed. $63 \%$ of the messages belonged to the category of irony and sarcasm, in accordance with the initial content of the rumour and its sense of humour.

The rumour was last spotted on the social media platforms a few days later, precisely on July 24 , 2016 on Facebook. Despite the enormous reaction rate reaching 10,113 interactions, it only persisted for a few days. The rumour only circulated for a month on Facebook. This may be related to the presence of official declarations concerning these economic reforms putting an end to the rumour.

\section{Rumour management/debunking}

\footnotetext{
7 Link to the rumor on Facebook, retrieved on April $30, \quad 2018 \quad$ : https://www.facebook.com/tora.for.u/photos/a.139055169499755/1157449647660297/?type=3\&theater ${ }^{8}$ Equivalent to $\$ 9$.
} 
The Da begad public page also played the role of fact-checking for this rumour and denied the rumour six days after it appeared on Facebook with screen shots of actual price tags of Pampers showing that prices didn't changed. The reach of this denial reveals its effectiveness in combating and curbing the spread of rumour. No announcement from $P \& G$ was officially published to deny the rumour.

\subsection{Orange Telecom}

\section{Rumour: «Orange is an Israeli company that sponsors Israeli military units».}

\section{Presentation of the case}

In 2015, Orange acquired Mobinil, an Egyptian mobile phone giant with the largest number of customers in Egypt. Before the announcement of the acquisition and the change of the company's name from Mobinil to Orange, a wave of rumours was launched on Facebook and Twitter. Boycott campaigns against the companies Mobinil and Orange were also conducted.

According to our observations, the public page called BDS Egypt on Facebook launched the rumour and spread it to Egypt on May 23, 2015. This rumour is considered a sensitive political matter for Egyptians since the state of war between the Arab Republic of Egypt and Israel, which dates back to the 1948-1949 Arab-Israeli war. Despite the signing of the Israeli-Egyptian peace treaty in 1978, the rate of investment and trade is still low: 117 Israeli organisations exported their products to Egypt in 2015 for a total amount of only US\$236 million (Darmon, 2016).

The spread of this rumour was characterised by numerous indexed hashtags present in the content of publications disseminating the rumour, in Arabic and French, as follows ${ }^{9}$ :

(translation: \#Egypt_boycott),

قاطع_موبينيل (translation: \#boycottMobinil),

مليونيه_مقاطعه شركات_الاتصالات (translation: One million telecommunication company boycotts), \#Orange-Āpartheid, \#Orange_Sanguine.

It is noteworthy that the design of the Arabic-indexed hashtags was similar to the jargons used in 2011 during the Arab Spring Revolution, which were designed to mobilise the masses. Using these indexed hashtags as keywords in the Facebook search section, twenty-five public pages on Facebook were found and were involved in spreading the rumour and linked to the BDS Egypt page. Of these Facebook pages, seven became inactive after an average of four months. These seven ephemeral pages played the role of a kind of astroturfing, a term that often describes actions taken by states or political groups to influence public opinion, the media or the vote as describes by Gautreau (2019). The political organisation BDS wanted to give the impression of a massively amplified boycott movement, when in fact it was orchestrated and coordinated by them alone.

Regarding the timing of the distribution of rumour publications, the BDS Egypt page followed a well-organized agenda that targets the hours when Egyptian Internet users are likely to be more active.

The photo launched by BDS Egypt, which caused the highest reaction rate, has been shared more than 2,000 times thanks to its design with little written content, the two logos of Mobinil and Orange, clearly visible and the Israeli flag in the middle of the page. It was launched on May 23, 2015 , accompanied by all the main hashtags of the boycott campaign.

\footnotetext{
${ }^{9}$ All hashtags were copied as they were launched originally even if they may contain some spelling mistakes.
} 
The reaction rate of Internet users to this publication on Facebook was marked by a volume of sharing that exceeded the volume of likes and comments.

The analyses of the messages, added before sharing the publication by Internet users, identify requests for a boycott of Orange.

During the propagation of this rumour, the existence of the account of a famous person in the circuit of the propagation reinforced the plausibility of the message transmitted. It was the account of an Egyptian politician named Mr. Sabahy- a former presidential candidate in Egypt and currently leader of a political party - began to support the attack campaign against Mobinil/Orange Egypt with a tweet containing the hashtags indexed from his certified account.

\section{Rumour management/debunking}

Two approaches have been adopted:

- Even if we are only interested in the Facebook platform, Orange's crisis management on Twitter deserves our attention. On Twitter, the company has been very active and has responded directly to comments from Internet users and BDS Egypt. The Orange Egypt community manager has given a lot of importance to Mr. Sabahy's Tweets. He responded to these tweets within ten minutes of their broadcast. Orange Egypt's interactive tempo shows that its reaction was the fastest when a famous person relayed the rumour than when the source came from other users.

- On Facebook, the public page Da begad took the initiative to post an official denial from Orange Egypt.

\section{The main findings and the analysis of the cases}

In this section, we identify the patterns that highlight the specificities of the dissemination of rumours in Egypt and the elements that feed them on Social media. To perform this, we will study the three cases presented through the multi-level analysis grid presented earlier.

\subsection{At the micro-environmental level}

Four variables are taken into consideration here.

- The beginning of the process of dissemination to the public.

Finding the person responsible for a rumour campaign is complicated. In our empirical research, the launching source of the rumour has been noticed to be isolated individuals, hidden behind public pages on Facebook; they have never used individual accounts. One of the members of the BDS organisation in Egypt, which sponsored the launch of the rumour against the Orange company, explained to us, during a semi-directive interview, the coordinated effort of several actors acting at different levels to effectively manage the sustainability of the rumour. Steps pretty similar to a publicity campaign.

- Frequency of the propagation 
According to our observations, the frequency of the dissemination of a rumour varies according to three situations.

- Rumour already has ancient roots on the Web. This was the case for Orange and Danone. The frequency with which these rumours are spread was cyclical throughout the years.

- Rumours intensify according to specific events: Ramadan for Danone yoghurts), the acquisition of Mobinil by Orange.

- The rumour is associated with the promulgation of a new economic law. This was the case for Procter \& Gamble when a new VAT was applied. Here, the rumour resonates with the mistrust, anxiety or even resistance to change of the members of a society.

- Involvement of celebrity accounts in the propagation process

This situation was only observed in the case of Orange, but only on the Twitter platform when certain "famous" individuals get involved in the rumour circuit, which can boost the effect of its propagation on social media.

\section{- Sentiment and semantic analysis of internet users}

It is important to note that the functionality of Facebook, allows internet users to add written texts and comments before sharing a publication which allows them to express their own feelings and opinions. This behaviour was observed in all three cases presented.

During the spread of the rumours studied in Egypt, three categories of feelings were observed: religious beliefs, the call for action to boycott (call for action), and sarcasm. For Danone, the messages added by Internet users are characterised by religious expressions such as "Allah is enough for me, he is the best guarantor". These religious expressions serve to express deep discontent and reflect the emotional state of the individuals concerned. For Pampers, the sentiment expressed is linked to sarcasm and/or a sense of humour. It is important to mention that this feeling was already embedded in the content of the rumour on Facebook. We deduce from this that the initial message used for the rumour conditions, at least partially, the reactions of Internet users.

\subsection{The meso-environmental level}

Here as well, four dimensions must be taken into account.

\section{- The form of the most widely disseminated rumour}

Our analyses reveal that, during the launch of a rumour, Internet users' reactions are particularly strong when photos are shared. This confirms that the spread of a rumour is partly motivated by visual messages. These photos contain transposable signs that evoke certain emotional emotions (such as a picture of a pig for a community who follows a religion that prohibits its consumption), which incite Internet users to spread the rumour.

\section{- Affective metrics reflecting reactions}

The aim here is to assess the nature of Internet users' engagement. According to our analyses, Egyptian Internet users had three reactions: sharing the publication, liking and writing comments. 
For Orange and Danone, we found that the first reaction was the most frequent. This observation contradicts the trends normally followed on Facebook where the likes rate exceeds the rate of sharing and comments ${ }^{10}$.

\section{- Rumour design: transposable signs and hashtags}

A wide variety of transposable signs were found: added symbols (e.g., a flag) or integrated elements (e.g., images). The content of the rumours also included indexed hashtags calling for boycotts, as in the case of Pampers and Orange. These various indexed hashtags, which were specifically created for these boycott campaigns, played a vital role in the rumour reach on social media. Hashtags are symbols that reinforce the sense of belonging to a group that defends a cause; this reinforces the credibility of rumours in the minds of Internet users.

\section{- Digital traceability and Web memory}

In the case of Orange for example, thanks to the digital traceability provided by the Web memory, new public pages on Facebook have been detected. Exactly twenty-five public pages were created to increase the spread of rumours by multiplying sources. This variable characterising the Web 2.0 was pivotal to help us conduct our data collection and through netnography and using data retrieval softwares such as Netvizz, NodeXl. And Gephi.

\subsection{Macro-environment level}

At this last stage of analysis, the four variables to be considered are as follows.

\section{- $\quad$ Topics and themes related to the cultural context}

The themes of the rumours studied are closely linked to the culture in Egypt. They concern health and religion (Danone), politics (Orange) and the economy (Pampers). Thanks to the three axes proposed by the sociologist Shibutani (1966), we were able to establish a link between the themes and the situational contexts that led to the emergence of rumours.

The first axis focuses on the relevance of situational context observed. For example, during the rumour about Pampers, it appeared just after the economic reforms announced in Egypt in 2016. The latter provided a favourable situational context to support the plausibility of the rumour. Another example, demonstrating the relevance of the situational context, concerns the rumour about Danone yoghurts just before Ramadan in a rather Muslim context ${ }^{11}$.

The second axis is concerned with the collective interpretation of a rumour. Rumours seem more plausible if there is a collective and shared meaning and consciousness. This triggers an emotion of belonging (emotional activation). This was observed with the rumour about the Orange

\footnotetext{
${ }^{10}$ Link to the study: https://www.quintly.com/blog/new-facebook-reaction-study. Accessed on 17 September 2018.

${ }^{11}$ In the Egyptian Constitution (URL link: https://www.constituteproject.org/constitution/Egypt_2014.pdf), according to article 2, "Islam is the religion of the State and Arabic its official language. The principles of the Islamic Shariah are the main source of the legislation. "The preamble states that "their interpretation derives from the jurisprudence of the Supreme Constitutional Court". Retrieved on February 16, 2019.
} 
company. The reaction of Internet users was to write comments before sharing the rumour on their Facebook walls.

The third axis reflects the degree of emotional activation (arousal). The emotions activated by the rumours studied are associated with the themes discussed and translated into the written messages. In the case of rumours based on a political theme, the messages called for a boycott action. Regarding the theme of health, Internet users expressed themselves through messages containing religious terms. For the economic theme, the messages were sarcastic to criticize the new laws. Rumours can thus create an emotional shock that encourages users to share frustration or anger on their Facebook walls.

\section{- language used during the interactions}

In the three cases studied, particularly during the appearance on Egyptian territory, the language used was Arabic with an Egyptian dialect. The interactions of Internet users, in the form of comments or messages added before the act of sharing, have used this language. With the exception of the rumours about Danone and Orange, since the traces on the digital platforms showed that they were launched from another territory before arriving to Egypt. These "extraterritorial" as we suggest to name them, have adapted the language and dialect to each country.

\section{- Cluster formation}

The data treated from the study data-set revealed the existence of cluster formation. This variable is well linked to culture since clusters appeared as social representations distributed in space and strongly linked to local/national values according to Latané's theory of dynamic social impact (1996).

According to our analysis, the public pages on Facebook that form the clusters are managed by people from different countries. The resulting cultural diversity thus reinforces the need for a communication style adapted to each cultural context.

\section{- $\quad$ Rumour management/debunking}

In the three cases, the rumours were denied by a verified public page on Facebook entitled $D a$ Begad? (ده بجد). This page, plays the role of a fact-checker, is followed by more than one million subscribers. It is important to note that the option, which exists on Facebook in different countries, to report that a piece of information is false is not active in Egypt. This option, proposed by the platform, invites users to signal false content in order to correct the information transmission algorithm. However, Egyptian Internet users have no reliable source other than the Da begad page to check the veracity of publications.

\section{Conclusion and modeling of the analysis grid}

Our observations and analysis suggest that the process of launching a commercial rumour is far from a natural or random phenomenon. To study the rumours in Egypt in depth, we proposed an analysis grid based on twelve variables at three levels: micro, meso- and macro-environment. This 
grid allows us to say that the initiators of rumours on social media carefully plan their entry through public accounts and pages. Internet users, by spreading false information, can be indeed considered as active stakeholders.

The twelve variables proposed to identify the process of spreading a rumour functioned as leverages to facilitate the achievement of certain goals, slandering the e-reputation of companies. Thus, with a minimum knowledge of the cultural particularities of a country, the act of propagation can have more or less long and more or less negative effects. By providing insights into the tactics used, our research aims to help companies understand this propagation phenomenon in order to better combat these attacks, which tend to increase in number.

We can conclude that rumours dissemination depends on socio-cultural factors. Ignoring the effects of these factors on Internet users' behaviour can discredit the image of companies on social media. To contextualise the applied analysis grid, we summarise it in (figure 14.1) as follows:

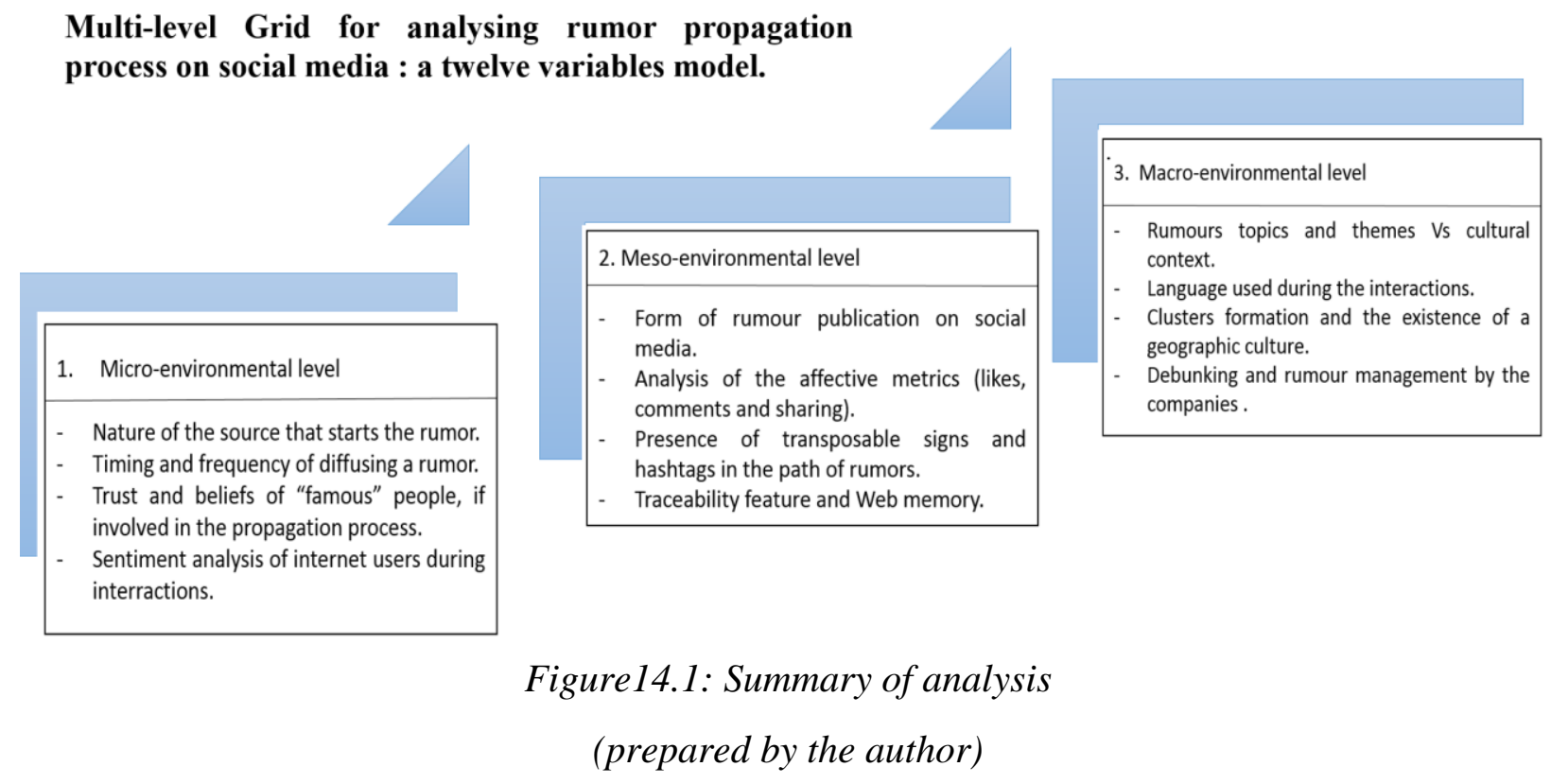

\section{REFERENCES}

Alloing C. (2013), «Processus de veille par infomédiation sociale pour construire l'e-réputation d'une organisation. Approche par agents-facilitateurs appliquée à la DSIC de La Poste ». Doctoral dissertation, Université de Poitiers.

Boulding, K. (1956), The image-knowledge in life and society. Ann Arbor: University of Michigan Press.

Brodin O., Roux E. (1990), Les recherches sur les rumeurs : courants, méthodes, enjeux managériaux. Recherche et Applications en Marketing (French Edition), Vol. 5, No. 4, P.45-70. https://doi.org/10.1177/076737019000500403 
Bruns A.; Burgess, J. E. (2011), The use of Twitter hashtags in the formation of ad hoc publics, in Proceedings of the 6th European consortium for political research (ECPR) general conference, University of Iceland, Reykjavik.

Colas F. (2004), Introduction au renseignement extérieur d'entreprise, Paris: l'esprit du livre éditions.

Darmon A. (2016, août 24), Hausse des investissements étrangers en Égypte, From Israël Export \& International Cooperation Institut, Israël Magazine. http://israelmagazine.co.il/hausseinvestissements-etrangers-Egypte/ (retrieved the $25^{\text {th }}$ of Octobre 2018).

Difonzo N. and Bordia P. (2007), Rumours influence: Toward a dynamic social impact theory of rumour The Science of Social Influence: Advances and Future Progress, A. R. Pratkanis (ed.), The Science of Social Influence, Philadelphia, PA: Psychology Press, 281-305.

Gautreau E. (2019), Expliquez-nous... L'astroturfing, retrieved from https://www.francetvinfo.fr/replay-radio/expliquez-nous/expliquez-nous-1 astroturfing_3119383.html on March 15, 2019.

Gerring J. (2004), What is a case study and what is it good for? American political science review, Vol. 98, N², P. 341-354.

Hall E. T. (1976), Beyond Culture, New York: Doubleday.

Kim C., Yang, S. U. (2017), Like, comment, and share on Facebook: How each behaviour differs from the other. Public Relations Review, Vol. 43, N², P. 441-449.

Kozinets R. V. (2002), The field behind the screen: Using netnography for marketing research in online communities. Journal of marketing research, Vol. 3, $\mathrm{N}^{\circ} 1$, p. 61-72.

Latané B. (1996), Dynamic Social Impact: The Creation of Culture by Communication, Journal of Communication, Vol. 46, $\mathrm{N}^{\circ} 4$, Autumn.

Le Béchec M. (2010), Territoire et communication politique sur le web régional breton, Doctoral dissertation, Université Rennes 2; Université Européenne de Bretagne.

Le Béchec M.? Boullier D. (2014), « Communautés imaginées et signes transposables sur un « web territorial » », Études de communication, Vol. 42 |2014, P. 113-126.

Milliot E. (2016), The Pivotal Role of Guanxi for Economic Intelligence in the People's Republic of China, Management international, Vol. 20, 4, P.133-145.

Le Béchec M.? Alloing C. (2018), Au-delà des traces numériques visibles, in Zlitni Sami, Liénard Fabien, Réseaux sociaux, traces numériques et communication électronique, Le Havre, 2018.

Ploux F. (2001), « Bruits alarmants et fausses nouvelle dans la France du XIXe siècle (18141870 », Hypotheses, Vol. 4, N¹, p. 303-314. 
Rouquette M. (1975), Les Rumeurs, Paris : PUF. P. 79-89.

Rouquette M. (1979), Les Phénomènes des Rumeurs, Thèse de doctorat en Psychologie. Université de Provence, p. 117.

Shibutani T. (1966), Improvised news: A sociological study of rumour. Indianapolis: The BobbsMerrill Company.

Stake R. (2006), Multiple Case Study Analysis. New York: The Guilford Press. 